\title{
Research on indoor visible light communication system using LiNbO3 thin film material as external modulator
}

\author{
Zhao Hua $^{1, \mathrm{a}}$, Lv Qing ${ }^{1, \mathrm{~b}}$, Li Shaohua ${ }^{2, c}$, Jin huilong ${ }^{1}$ \\ 1、Hebei Normal University, Career Technical College, China, Hebei \\ Shijiazhuang, 050024; 2. The first hospital of Hebei Medical \\ University, China, Hebei, Shijiazhuang, 050031)

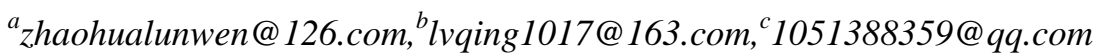

\begin{abstract}
Indoor visible light communication, as an emerging broadband wireless access technology, is currently in the stage of scientific research. This paper focuses on researching fractionally spaced equalizer for indoor visible light communication system, and studies have shown that equalization of indoor visible light communication "multipath interference" to the quality of the communication interference had good inhibitory effect. This paper also proved that the phase conjugate light and transmission light which generated by four wave mixing effect of LiNbO3 thin film materials have bright quantum entanglement properties. Using visible light communication system with nonlinear film material external modulator, it will be expected to realize indoor quantum communication.
\end{abstract}

Keywords: visible light communication; fractionally spaced; equalization; quantum communication

\section{Introduction}

Indoor visible communication system model uses the light LED array as a signal transmission source and the signal is unipolar, and adopted intensity modulation / direct detection (IM / DD) as the way of modulation and demodulation. White light signals emitted by the LED lighting are made the intensity-modulation in the signal transmitter (the signal light has an instantaneous power, as same as the binary " 1 ", no time, and then " 0 " is the same). The modulated visible light signals at different optical transmission path is transmitted to the signal receiving end, receiving terminal will do direct detection for the received signal, and decode related optical power information so as to export the transmission information . 


\section{Visible light communication System model using external modulator}

At present, visible light communication system in the near future is shown in figure 1.

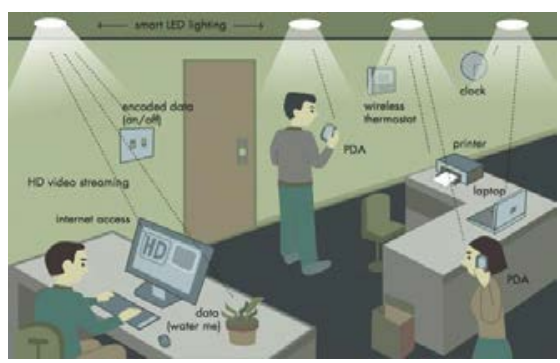

Fig.1 Indoor visible light communication system model

In this paper, the visible light signal modulation is studied, which is using $\mathrm{LiNbO}_{3}$ crystal that produced as the external modulator.

In this system, the relevant signals mathematically expressed as follows:

In the time domain, the indoor and ambient visible light communication signal transmission of visible light can adopt the corresponding real channel impulse function c (t) to describe ${ }^{[1]}$.

Mathematically, the visible light signal received by the signal receiver can be represented by $\mathrm{r}(\mathrm{t})$, emitted light signal can be represented by $\mathrm{x}(\mathrm{t})(\mathrm{x}(\mathrm{t})$ of non-negative), a channel of optical noise can be represented by $\omega(t)$, signal convolution can be represented by $\otimes$.

The relationship between them is:

$$
r(t)=x(t) \otimes_{c(t)+\omega(t)}
$$

In the process of signal transmission, channel noise consists of shot noise and amplifier noise in receiver. Relevant scientific research shows that, in optical communications, on the case of the presence of channel noise, the formula of visible light signal above can be expressed as: in the formula (1) , $\mathrm{T}$ is the circle symbol, represents data input symbol in time n, Lk represents a channel length.

\section{FSE balancing algorithm}

Before adopting FSE channel equalization algorithm to calculate the optical channel, optical receiver will sample the output channel. Sampling process be simplified, $\mathrm{T} / \mathrm{L}$ of the $\mathrm{k}$-th sampling time period represented in rk. In the above formula $\mathrm{x}(\mathrm{nT})$ with $\mathrm{x}(\mathrm{n})$ represents, c (t-nt) = c (kT / L-nT) by ck, n represents, $\omega(\mathrm{t})=\omega(\mathrm{kT} / \mathrm{L})$ is represented by $\omega \mathrm{k}$. Therefore, using a time signal $\mathrm{k}$ can be expressed as: 


$$
\mathrm{r}(\mathrm{k})=\sum_{n=1}^{\mathrm{L}_{k}-1} x_{n} c_{k, u}+\omega_{\mathrm{k}}
$$

After the signal is acquired, it needs to make a filtering process, which requires to send the signal to the FIR equalizer, an equalizer tap interval of $\mathrm{T} / \mathrm{L}$, the signal finishes being filtered needs to be extracted before being outputted by decimation $\mathrm{L}$.

\subsection{Balancing Algorithm}

In the FIR equalizer, the convolution between the sample sequence and the weight coefficient of equalizer can be expressed sequences of the filtering process by the equalizer, therefore, the output signal of the equalizer can be expressed as:

$$
S_{k}=\sum_{i=1}^{L \bullet N_{f}-1} f_{i} r_{k-i}
$$

Where, $f_{\mathrm{i}}$ is fractionally spaced equalizer weights, $N_{\mathrm{f}}$ is the equalizer symbols, $L \cdot N_{\mathrm{f}}$ is the equalizer length.

A transmission signal of the equalizer for the sample, sampling the output signal is expressed as:

$$
y_{n}=s_{L n}=\sum_{i=1}^{L \bullet N_{f}-1} f_{i} r_{L n-1}
$$

The (2) into (4), the matrix expression is obtained sampled output signal:

$$
\mathrm{y}(\mathrm{n})=(\mathrm{xT}(\mathrm{n}) \mathrm{C}+\mathrm{wT}(\mathrm{n})) f
$$

\subsection{FSE coefficient optimization}

When designing the equalizer, using the MMSE criterion, therefore, for the systematic error, the followings will be in accordance with the following formula.

$$
\mathrm{e}(\mathrm{n})=\mathrm{y}(\mathrm{n})-\mathrm{x}(\mathrm{n}-\delta)
$$

Where, $\delta$ is the error in the calculation of the variable, which can be optimized.

The (5) into (6), we obtain a formula for the error:

$$
\mathrm{e}(\mathrm{n})=\mathrm{xT}(\mathrm{n})(\mathrm{Cf}-\mathrm{h} \delta)+\mathrm{wT}(\mathrm{n}) f \text {. }
$$

Analyzing the formula (7), using the method of source normalization, FSE coefficient minimum, the minimum mean square error, the equalizer coefficient vector and the best delay required to meet the optimization of publicity $\mathrm{f}=$ $\mathrm{A}-1 \mathrm{CHh} \delta$, to optimize the results, we know that the minimum mean square error: 


$$
J_{M S E}=h_{\delta}^{H}\left(I-C A^{-1} C^{H}\right) h_{\delta}
$$

\section{3 computational analyses}

An algorithm for calculating the amount of the process is not very precise evaluation of the data, and the evaluation methods used in the computation algorithm is typically bound on the number of basic operations evaluated. In this paper, in order to calculate the $\mathrm{N}$-point signal input through the fractionally spaced equalizer output after the number of multiplications required to calculate the amount to account for the size, the size of the amount calculated by using the $\mathrm{O}$ to measure. Calculating the multiplication by using the FSE method is $\mathrm{O}\left(\mathrm{LN}_{2}\right)$ + $\mathrm{O}\left(L_{3} N f_{3}\right)$, the multiplication calculation of the MMSE algorithm in $\mathrm{O}\left(N_{2}\right)+$ $\mathrm{O}\left(L f_{3}\right)$. From the analysis of the above formula, the FSE algorithms to perform a calculation are related of the calculation amount and the size and length of the equalizer sampling interval, and the calculation with two variables constitutes the polynomial. Therefore, a reasonable amount of computation balancing algorithm used in this paper, in practice is feasible.

\section{Simulation analysis}

In this paper, computer simulation technology is adopted to evaluate FSE equalization algorithm, and the evaluation index is used in the bit error rate (BER) and mean square error (MSE).

Linear relationship (OSNR) between the following picture two and three, respectively, the following figure shows the two equalizers MSE and BER performance and optical signal to noise ratio.

As can be seen from the two figures, the spacing of the linear channel equalizer to compensate for inter symbol interference caused by the communication quality does not completely eliminate, but the fractionally spaced equalizer is able to better compensate for the channel distortion of the visible light communication and to suppress inter-symbol interference. 


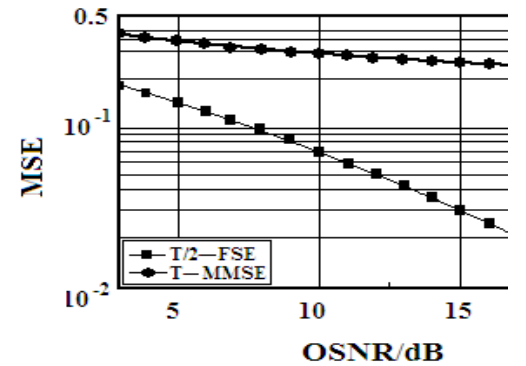

Fig. 9a OSNR-MSF performance curves

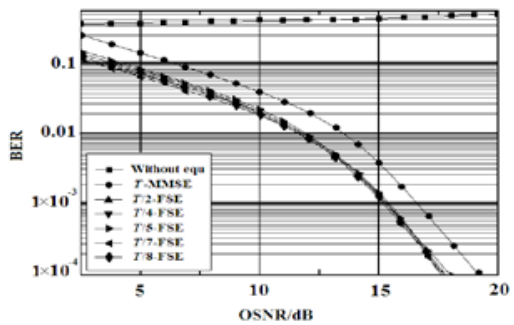

Fig. 9b OSNR-BER performance curves under two equalization method different sampling intervals

\section{The four wave mixing effect of visible light communication when using external modulator made by the new $\mathrm{LiNbO}_{3}$ thin Film material.}

In 1935, Einstein, Podolsky and Rosen (EPR) in an attempt to demonstrate the incompleteness of quantum mechanics, put forward a two particle entangled state, namely the EPR state ${ }^{[2]}$ :

$$
\left|\psi>=\sum \delta\left(\mathrm{a}+\mathrm{b}-\mathrm{c}_{0}\right)\right| \mathrm{a}>\mid b>\ldots \ldots \ldots \ldots \ldots \ldots \ldots \ldots \ldots(9)
$$

When study the nonlinear optical effect of the new $\mathrm{LiNbO}_{3}$ thin film material, the phase conjugate light and transmission light has been found have the entangled state. Further experiments will be used to validate the four wave mixing effect when the visible lights transmit the new material of $\mathrm{LiNbO}_{3}$ thin film. The experimental design can use 488nm argon atom laser as the light source

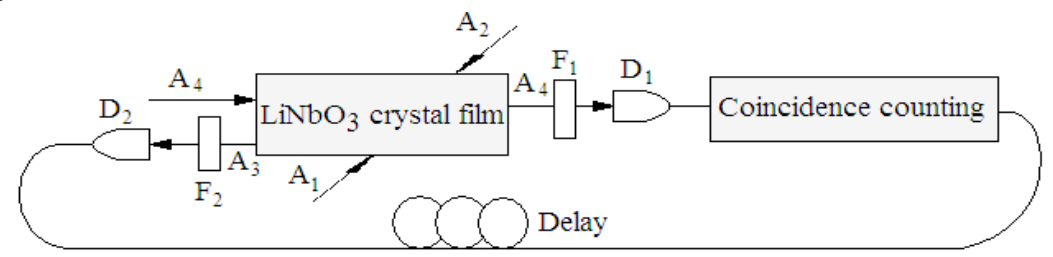

Fig.10 Principle of measurement of entangled photon pairs produced by four waves mixing in $\mathrm{LiNbO}_{3}$ thin film

Visible light communication system using white LED as backlight (carrier light source), so there is a lot of kinds of Wavelength in the optical carrier, the formation of four waves mixing in $\mathrm{LiNbO}_{3}$ film modulator are easy ${ }^{[3,4]}$.

The principle of measurement of entangled photon pairs which are produced by the degenerate four waves mixing is shown as the figure10. The $D_{1}$ 、 $\mathrm{D}_{2}$ is signal detector, respectively used to receive the phase conjugation light and transmission light, $F_{1} 、 F_{2}$ is the filter, which is ensure the stray light cannot enter the detector, and make $\mathrm{Z}$ direction as the horizontal direction. 
As the pumping light field is very strong, it can be treated as the classical case:

$$
\begin{aligned}
& \mathrm{E}_{\mathrm{i}}^{(+)}(\mathrm{r}, \mathrm{t})=\int_{-\infty}^{+\infty} \mathrm{d} \omega\left(\mathrm{A}_{\mathrm{i}}(\omega) \mathrm{e}^{\mathrm{i}\left(\omega \mathrm{t}-\mathrm{k}_{\mathrm{i}} \cdot \mathrm{r}\right)}+\right. \\
& A_{i}^{*}(\omega) \mathrm{e}^{-j\left(\omega t-k_{i} \cdot r\right)}
\end{aligned}
$$

In the formula (10), $\mathrm{i}=1,2, \mathrm{r}$ is a position vector, parallel to the direction of the pump light, $\mathrm{Ai}(\omega)$ is the spectrum distribution function of pumping light field, general is Gauss type, its expression as follows:

$$
\mathrm{A}_{\mathrm{i}}(\omega)=\mathrm{A}_{\mathrm{i}} \exp \left[-\frac{(\omega-\Omega)^{2}}{2 \delta_{\mathrm{p}}^{2}}\right]
$$

In the formula(11), $\omega$ is the center frequency of the pump, $\delta_{\mathrm{p}}$ is half high width, The quantization of signal light field and conjugate optical field can be expressed as follows:

$$
E_{j}^{(+)}=\sum_{k} E_{j k} \hat{a}_{j k} e^{i\left(k \cdot r-\omega_{j k} t\right)}
$$

In the formula(12), $\mathrm{j}=3,4, \hat{a}_{\mathrm{jk}}$ is corresponding to the annihilation operator which polarization is $\mathrm{j}$ and the wave vector is the mode of $\mathrm{k}$ 。 And $\hat{\mathrm{a}}_{\mathrm{jk}}$ satisfy the commutation relations : $\left|\hat{\mathrm{a}}_{\mathrm{jk}}, \hat{\mathrm{a}}_{\mathrm{mq}}^{+}\right|=\delta_{\mathrm{jm}} \delta_{\mathrm{kq}}$, among them, $\hat{a}_{\mathrm{mq}}^{+}$corresponding to the generated operator which polarization is $\mathrm{m}$ and wave vector is mode of $\mathrm{q}$; dispersion relation of medium is $|\mathrm{k}|=\frac{\omega_{\mathrm{ik}} \mathrm{n}_{\mathrm{jk}}}{\mathrm{c}}, \mathrm{n}_{\mathrm{jk}}$ is the refractive index; $\mathrm{E}_{\mathrm{jk}}=\mathrm{i}\left\{\frac{\square \omega_{\mathrm{jk}}}{2 \varepsilon_{0} \mathrm{n}_{\mathrm{jk}} \mathrm{V}_{\mathrm{Q}}}\right\}^{\mathrm{V}^{2}}, \square$ is Planck's constant, $\varepsilon_{0}$ is the vacuum dielectric constant, VQ is the quantized volume ${ }^{[5,6]}$. In the context of interaction, The Hamiltonian of four beam of light for the interaction is:

$$
\mathrm{Hi}=\varepsilon_{0} \int_{\mathrm{V}} \mathrm{d}^{3} \mathrm{r}\left(\mathrm{x}^{(3)} \mathrm{E}_{1}^{(+)} \mathrm{E}_{2}^{(+)} \mathrm{E}_{3}^{(-)} \mathrm{E}_{4}^{(-)}+\mathrm{x}^{(3) *} \mathrm{E}_{4}^{(+)} \mathrm{E}_{3}^{(+)} \mathrm{E}_{2}^{(-)} \mathrm{E}_{1}^{(-)}\right) \ldots \ldots \ldots \ldots \ldots
$$

Wherein, $\mathrm{x}(3)$ is the three order nonlinear coefficient of the $\mathrm{LiNbO}_{3}$ film. $\mathrm{V}$ is the volume which is Illuminated by the pump light, $\mathrm{E}(-)$ and E (+) indicates Hermitian conjugate. Considering only the $\mathrm{Z}$ component for the signal light and transmission light, there $\mathrm{k}_{3}=\mathrm{k}^{\prime} \hat{\mathrm{e}}_{\mathrm{z}}, \mathrm{k}_{4}=\mathrm{k}^{\prime \prime} \hat{\mathrm{e}}_{\mathrm{z}}, \hat{\mathrm{e}}_{\mathrm{z}}$ is the unit vector along the $\mathrm{Z}$ direction $\mathrm{k}^{\prime}=\left|\mathrm{k}_{3}\right|, \mathrm{k}^{\prime \prime}=\left|\mathrm{k}_{4}\right|$. Based on the first-order perturbation theory, the system state is:

$$
\begin{gathered}
|\psi>=| 0> \\
-\frac{i}{\hbar} \int_{-\infty}^{\infty} \mathrm{d} t \mathrm{H}_{\mathrm{I}}|0>=| 0>+\iint \mathrm{d} \omega_{1} \mathrm{~d} \omega_{2} A\left(\omega_{1}\right) A\left(\omega_{2}\right) \int_{\mathrm{z}} \mathrm{dz} \sum_{k^{\prime} k^{\prime \prime}} \mathrm{F}_{\mathrm{k}^{\prime} \mathrm{k}^{\prime \prime}} \hat{\mathrm{a}}_{3 \mathrm{k}^{\prime}}^{+} \hat{\mathrm{a}}_{4 \mathrm{k}^{\prime \prime}}^{+}
\end{gathered}
$$

The function $\mathrm{F}_{\mathrm{k}^{\prime} \mathrm{k}^{\prime \prime}}=\Gamma_{\mathrm{k}^{\prime} \mathrm{k}^{\prime \prime}} \delta\left(\omega_{1}+\omega_{2}-\omega_{3}-\omega_{4}\right) \exp \left(-\mathrm{iz} \Delta_{k^{\prime} k^{\prime \prime}}\right), \Delta_{\mathrm{k}^{\prime} \mathrm{k}^{\prime \prime}}=k_{1}+$ $\mathrm{k}_{2}-\mathrm{k}_{3}-\mathrm{k}_{4}$ indicates the phase mismatch, $\Gamma_{\mathrm{k}^{\prime} \mathrm{K}^{\prime \prime}}=\frac{-1}{\hbar} \mathrm{E}_{3 \mathrm{k}^{\prime}} \mathrm{E}_{4 \mathrm{k}^{\prime \prime}} 2 \pi \varepsilon_{0} \mathrm{X}^{(3)} \mathrm{S}, \mathrm{S}$ is the cross-sectional area which interact each other, and which are illuminated by 
pump light. The formula (14) also contains $\delta(\cdot)$, the same structure with formula (9), namely the two-photon entangled state.

Therefore, the phase conjugate light and transmission light produced by four wave mixing experiments using the new $\mathrm{LiNbO}_{3}$ thin film materials is the light in quantum entanglement. The nonlinear coefficient of thin film materials is larger, so the generated two-photon entangled with higher strength ${ }^{[7,8]}$. A new method of preparation for high power bright entangled photon source is provided by using nonlinear thin film materials and the experimental method in this paper.

\section{Conclusion}

$\mathrm{LiNbO}_{3}$ thin film materials which grown on $\mathrm{SrTiO}_{3}$ substrate is a new type of transparent material. It has good selectivity of visible light, and can be used for preparing the visible light signal transmitter external modulator. The new type $\mathrm{LiNbO}_{3}$ thin film materials can provide hardware support for the equalization of indoor visible light communication signals. At the same time, the four wave mixing effect in the new type of $\mathrm{LiNbO}_{3}$ thin films can be used in preparation of bright entangled photon pairs, so it has wide application prospects in the field of quantum communication.

\section{Acknowledgements}

This work was financially supported by the Hebei Province Education Office Foundation (N2014116), Hebei Normal University Youth Foundation (L2010Q10), and Hebei Normal University Doctoral Foundation (L2012B15).

\section{Reference:}

[1] M. Veithen and Ph. Ghosez. First-principles study of the dielectric and dynamical properties of lithium niobate[J]. Phys. Rev. B, 2002, 65(21): 214302-1-12

[2] Einstein A, Podolsky B, Rosen N. Can quantum-mechanical description of physical reality be considered complete?[J].Phy.Rev.,1935,47(10):777-780.

[3] C. Ederer and N. A. Spaldin. Effect of epitaxial strain on the spontaneous polarization of thin film ferroelectrics [J]. Phys. Rev. Lett., 2005, 95(25): 257601-1-4

[4] H. T. Li, Y. D. Xia, B. Xu, et al. Memristive behaviors of LiNbO3 ferroelectric diodes[J]. Appl. Phys. Lett., 2010, 97(1): 012902-1-3 
[5] H. X. Xu, A. Chernatynskiy, D. Lee, et al. Stability and charge transfer levels of extrinsic defects in LiNbO3[J]. Phys. Rev. B, 2010, 82(18): 184109-1-8

[6] J. Y. Dai, H. K. Lam, Quan Li, et al. Structural characterization and electron-energy-loss spectroscopic study of pulsed laser deposited $\mathrm{LiNbO}_{3}$ films on a-sapphire [J]. J. Appl. Phys., 2004, 96(11): 6319-6322

[7] D. V. Roshchupkin, D. V. Irzhak, and V. V. Antipov. Study of $\mathrm{LiNbO}_{3}$ and $\mathrm{LiTaO}_{3}$ ferroelectric domain structures using high-resolution x-ray diffraction under application of external electric field [J]. J. Appl. Phys., 2009, 105(2): 024112-1-6

[8] I. Inbar and R. E. Cohen. Comparison of the electronic structures and energetic of ferroelectric $\mathrm{LiNbO}_{3}$ and $\mathrm{LiTaO}_{3}$ [J]. Phys. Rev. B, 1996, 53(3): 1193-1203 